\title{
NLTE barium abundance in thin and thick disks of the Galaxy
}

\section{S. Korotin ${ }^{* \dagger}$}

Astronomical Observatory of Odessa National University, Ukraine

E-mail: serkor@skyline.od.ua

\section{T. Mishenina, T. Gorbaneva}

Astronomical Observatory of Odessa National University, Ukraine

E-mail: tamardeneb1.odessa.ua

\section{Soubiran}

Observatoire Aquitain des Sciences de l'Univers, Floirac, France

E-mail: soubiran@observ.u-bordeaux.fr

We present the determinations of the barium abundance taking into account the non-LTE (NLTE) effects in 173 dwarf stars belonging to the thin and thick disks. The NLTE profiles of the unblended Ba II lines (4554, 5853, $6496 \AA \AA$ A) have been computed and compared to the observed profiles. The line $6141 \AA$ was also used, but taking into account an influence of iron line. We used a modified version of the MULTI code and atomic model of Ba atom with 31 levels of Ba I, and 101 levels of Ba II. Atmosphere models of investigated stars have been calculated with ATLAS9 code (modified using the New Opacity distribution functions by Castelli \& Kurucz). The obtained trend in the $\mathrm{Ba}$ abundance vs. $[\mathrm{Fe} / \mathrm{H}]$ suggests a complex process of the Ba production in the thin and thick disks.

11th Symposium on Nuclei in the Cosmos

19-23 July 2010

Heidelberg, Germany.

* Speaker.

${ }^{\dagger}$ This work was supported by the Swiss National Science Foundation (SCOPES project No.IZ73Z0-128180/1) 


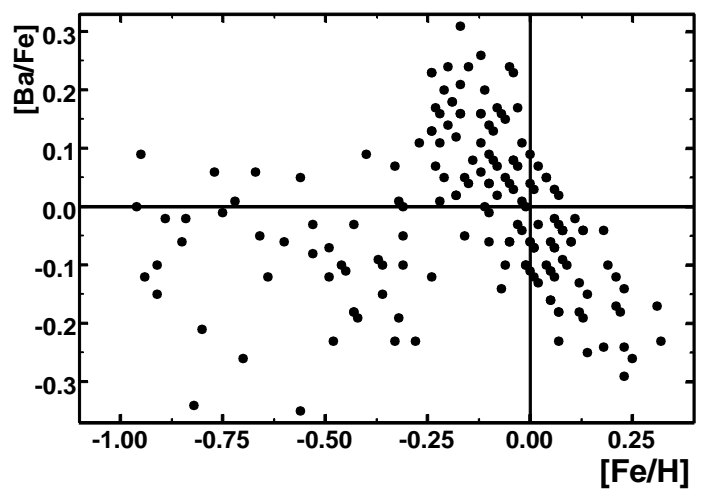

Figure 1: LTE Ba abundance vs. Fe

The distribution of each chemical element abundance over the Galaxy is important for the recovery of the picture of its chemical evolution. The behavior of the barium abundance within the metallicities from -1.0 to +0.3 is of the great interest because the observations show the absence of the smooth dependence upon metallicity (the presence of a "jog", Bensby et al. [1]). As known, the derived barium abundance is significantly affected by the NLTE effects (Mashonkina et al. [2], Andrievsky et al. [3]). Particularly it is true for metal-poor stars.

The spectra of 172 stars were obtained in the region of 4400-6800 $\AA$ with S/N about 100-350 using the 1.93-m telescope at Observatoire de Haute-Provence (OHP, France) equipped with the echelle-spectrograph ELODIE (Baranne et al. [4]) which provides a resolving power of $\mathrm{R}=42000$.

The selection of the stars was made on kinematic criterion using probability of the star belonging to the thin, thick disk and Hercules stream, based on spatial velocity of a star and kinematic parameters of the disks (Mishenina et al. [5], Soubiran et al. [6]).

Atmospheric parameters were determined earlier in our work (Mishenina et al., [5]).

First of all the barium was determined in LTE using only one line $4554 \AA$ (Fig 1.) The shape of obtained dependence between $[\mathrm{Ba} / \mathrm{Fe}]$ and $[\mathrm{Fe} / \mathrm{H}]$ appeared to be strange. After that we used four lines to derive barium abundance $(4554,5853,6141$ and $6496 \AA \AA \AA)$ in the NLTE approximation.

Our barium model contains 31 levels of Ba I, 101 levels of Ba II with $\mathrm{n}<50$, and the ground level of Ba III ion. In the detailed consideration we included 91 bound-bound transitions.

A cause of some uncertainty in the NLTE analysis of the barium spectrum is the scarce information about the photoionization cross-sections for the different levels. We used the results of the scaled Thomas-Fermi method application (Hofsaess [7]).

Effective excitation electron collisional strengths for the transitions between the first levels $\left(6 s^{2} \mathrm{~S}, 5 \mathrm{~d}^{2} \mathrm{D}\right.$ and $\left.6 \mathrm{p}^{2} \mathrm{P}^{0}\right)$ were used following Schoening \& Butler [8]. Experimental cross-sections for the transitions $6 s^{2} S-7 s^{2} S$ and $6 s^{2} S-6 d^{2} D$ were taken from Crandall et al. [9]. Collisional rates for the transitions between sublevels $5 \mathrm{~d}^{2} \mathrm{D}, 6 \mathrm{p}^{2} \mathrm{P}^{0}$ and $7 \mathrm{~s}^{2} \mathrm{~S}, 6 \mathrm{~d}^{2} \mathrm{D}$, and between $7 \mathrm{~s}^{2} \mathrm{~S}$ and $6 d^{2} D$ were estimated with the help of the corresponding formula from Sobelman et al. [10]. For the rest of the allowed transitions, we used the van Regemorter [11] formula, while for the forbidden transitions the Allen's [12] formula was used. The rate of the collisional ionization from the ground level of Ba II was calculated using the corresponding formula in Sobelman et al. [10]. 


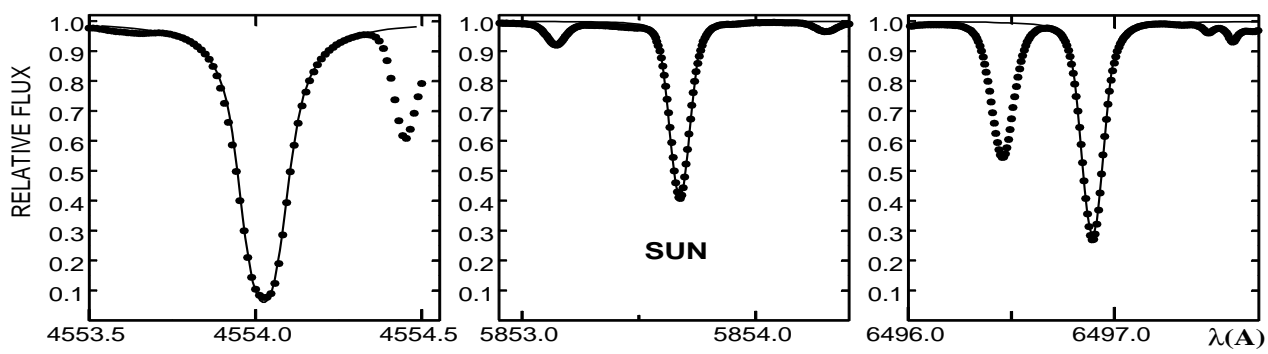

Figure 2: Profile fitting in the solar spectrum.
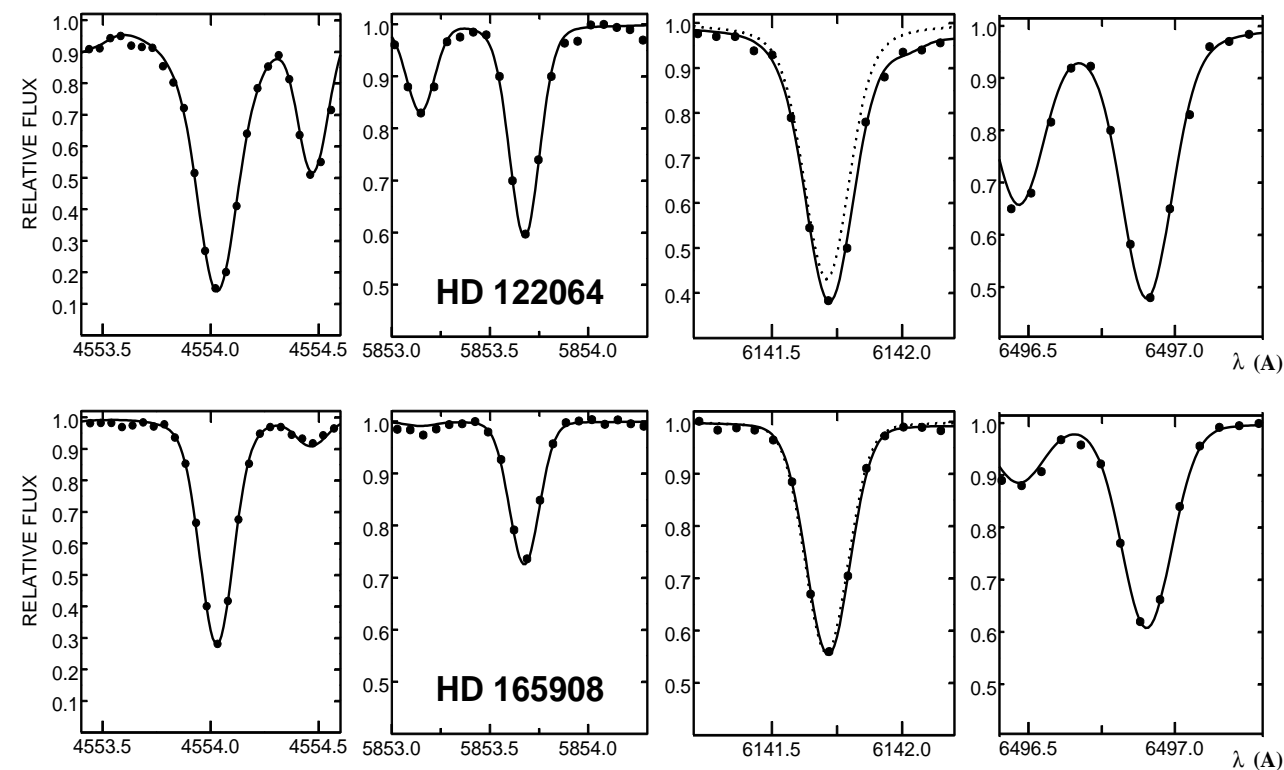

Figure 3: Profile fitting in the stars.

The NLTE profiles of the barium lines were computed using a modified version of the MULTI code (Carlsson [13]). The modifications are described in Korotin et al. [14].

The odd barium isotopes have hyper-fine splitting of their levels and thus several HFS components for each line. Therefore, the line $4554 \AA$ was fitted in the solar spectrum (Fig. 2) by adopting the even-to-odd abundance ratio of 82:18 (Cameron [15]). The solar abundance of barium 2.17.

The line $6141 \AA$ is blended with the iron line (its contribution can be traced in Fig. 3; the dot line - is the barium line profile).

As a result of NLTE analysis application the shape of the $[\mathrm{Ba} / \mathrm{Fe}]$ vs. $[\mathrm{Fe} / \mathrm{H}]$ dependence changed. In Fig. 4: thin disk - solid circles, thick disk - open circles, Hercules stream - crosses, open squares - other. Thus, one can state that the LTE analysis of only one line $4554 \AA$ leads to systematic errors.

In the work of Serminato et al. [16] the barium abundance evolution was considered within the wide range of metallicity (taking into account the $\mathrm{s}$ - process contribution from AGB stars and 

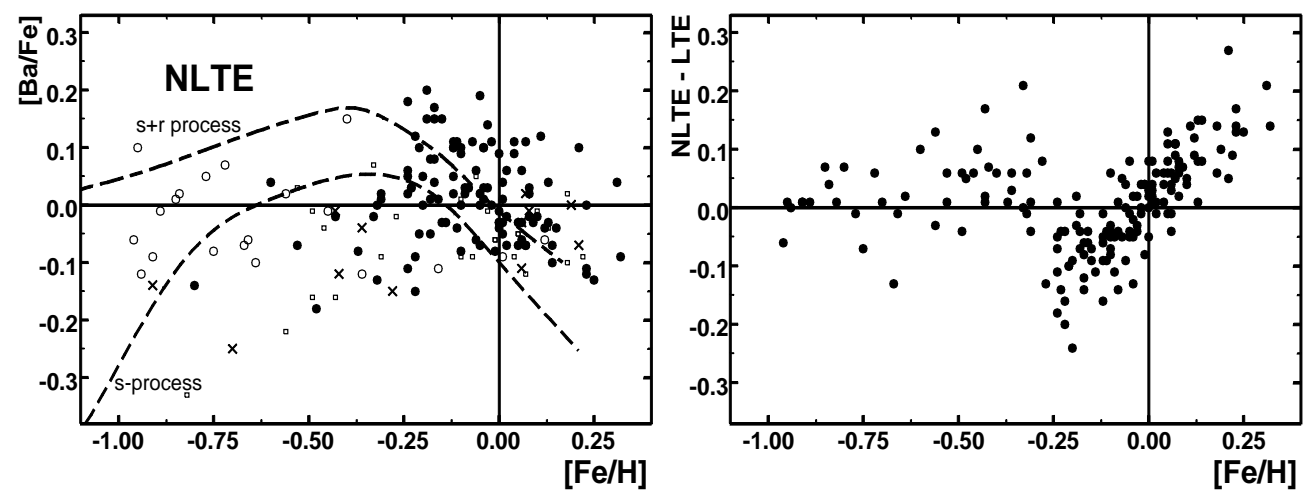

Figure 4: NLTE barium abundnace and NLTE corrections vs. metallicity.

stars with 8-10 solar masses). In Fig. 4 the upper curve takes into account the common contribution from $\mathrm{s}$ - and $\mathrm{r}$ - process, while lower line corresponds to $\mathrm{s}$ - process only.

As one can see theoretical calculations of the $r$ - process enrichment overestimate the $[\mathrm{Ba} / \mathrm{Fe}]$ ratio.

\section{References}

[1] T. Bensby, S. Feltzing, I. Lundstrom, I. Ilyin, $A \& A(2005)$ 433, 185

[2] L. Mashonkina, T. Gehren, I. Bikmaev, $A \& A(1999)$ 343, 519

[3] S.M. Andrievsky, M. Spite, S.A. Korotin et al., A\&A (2009) 494, 1083

[4] A. Baranne, D. Queloz, M. Mayor et al., A\&AS (1996) 119, 373

[5] T.V. Mishenina, C. Soubiran, V.V. Kovtyukh, S.A. Korotin, $A \& A(2004)$ 418, 551

[6] C. Soubiran, F. Girard, $A \& A(2005)$ 438, 139

[7] D. Hofsaess, $A D N D T(1979)$ 24, 285

[8] T. Schoening, K. Butler, $A \& A S(1998)$ 128, 581

[9] D.H. Crandall, G.H. Dunn, A. Gallagher et al., $A p J(1974)$ 191, 789

[10] I.I. Sobelman, L.A. Vainshtein, E. Yukov, Excitation of Atoms and Broadening of Spectral Lines, Berlin, Springer 1981

[11] H. van Regemorter, $A p J(1962)$ 136, 906

[12] C.W. Allen, Astrophysical Quantities, London: Athlone Press, 1973

[13] M. Carlsson, Uppsala Obs. Rep.(1986) 33

[14] S.A. Korotin, S.M. Andrievsky, R.E. Luck, $A \& A(1999)$ 351, 168

[15] A.G.W. Cameron, $\operatorname{Ap\& SS(1982)~82,123}$

[16] A. Serminato, R. Gallino, C. Travaglio et al., PASA(2009) 26, 153 